\title{
Manejo odontológico integral de paciente con antecedentes de ictus cerebral. Reporte de un caso
}

\author{
Comprehensive dental management of patients with a history of cerebral stroke. Report of a case
}

María Alejandra Puerta-Domínguez ${ }^{*}$ orcid.org/0000-0002-0831-8158

Isabella Manzur-Villalobos' ${ }^{1}$ orcid.org/0000-0003-1979-7454

Ana Isabel Llera-Ladera² orcid.org/0000-0002-7738-7077

Héctor Juan Rodríguez-Casanovas² orcid.org/0000-0003-4065-4538

Antonio Díaz-Caballero3 orcid.org/0000-0001-9693-2969

1 Joven Investigadora, Universidad de Cartagena. Cartagena, Colombia.

2 Facultad de Odontología, Universidad de Sevilla. Sevilla, España

3 Facultad de Odontología, Universidad de Cartagena. Cartagena, Colombia

\section{Resumen}

Introducción: Ictus son un conjunto de patologías que afectan a los vasos sanguíneos que suministran la sangre al cerebro produciendo una alteración de la función de una determinada región de éste. El grupo de patologías son conocidas popularmente como embolias o accidentes cerebrovasculares (ACV) y se manifiestan súbitamente. Estos trastornos tienen en común su presentación brusca y suelen afectar a personas mayores, aunque también pueden presentarse en jóvenes. Objetivo: Dar a conocer a los profesionales de la salud las implicaciones y consecuencias que se deben tener en la práctica odontológica habitual y al momento de emplear tratamientos como la rehabilitación protésica fija o la colocación de implantes dentales en pacientes con cardiopatías, con la finalidad de evitar accidentes y prevenir hemorragias postoperatorias. Resultados: Se obtuvo resultados estéticos y funcionales satisfactorios en los tratamientos quirúrgicos y de rehabilitación bucal realizados al paciente. Conclusiones: Debido al riesgo de presentar accidentes cerebrovasculares normalmente están sometidos a terapia antiagregante plaquetaria, cuyo efecto secundario es la tendencia al sangrado; por ello es importante realizar un manejo multidisciplinario con el médico especialista al momento de emplear tratamientos quirúrgicos en este tipo de pacientes, con el fin de evitar complicaciones y obtener resultados adecuados.

Palabras clave: Cardiopatías; accidente cerebrovascular; rehabilitación bucal; implantes dentales. (Fuente: DeCS, Bireme).

\begin{abstract}
Introduction: Stroke are a set of pathologies which affect the blood vessels that supply the blood to the brain causing an alteration of the function of a certain region. The group of pathologies are popularly known as embolisms or strokes and are suddenly manifested. These disorders have in common their abrupt presentation and usually affect older people; although, they can also be presented in youth. Objective: To introduce to health professionals the implications and consequences that should be taken in the habitual dental practice and at the time of using treatments such as fixed prosthetic rehabilitation or the placement of dental implants in patients with heart disease, in order to avoid accidents and prevent postoperative hemorrhages. Results: Satisfactory esthetic and functional results were obtained in the
\end{abstract}


surgical and oral rehabilitation treatments carried out to the patient. Conclusions: Due to the risk of stroke, the patients are usually subjected to antiplatelet therapy, whose side effect is the tendency to bleed. Therefore, it is important to carry out a multidisciplinary management with the specialist doctor when using surgical treatments in this type of patients in order to avoid complications and obtain adequate results.

Key words: Heart diseases; stroke; mouth rehabilitation; dental implants. (Source: DeCS, Bireme).

\section{Introducción}

Los odontólogos a lo largo de la práctica profesional tienen que enfrentarse a pacientes que presenten patologías sistémicas agudas o crónicas, que necesitan particular atención odontológica y se requiere tener conocimientos de las terapias específicas para que sean manejados de una manera adecuada, como es el caso del paciente con ictus cerebral. El ictus es un trastorno brusco de la circulación cerebral que altera la función de determinada región del cerebro; corresponde a un grupo de trastornos que tienen en común su presentación brusca, su epidemiologia es variable entre los diferentes países y entornos, y está en relación con factores genéticos, ambientales, la edad de la población y la prevalencia de otros factores de riesgo asociados(1). Suelen afectar a personas mayores aunque en un menor porcentaje también se ven afectados los jóvenes, se presenta más en hombres que en mujeres(2). Los ictus pueden presentarse por una disminución del flujo sanguíneo que recibe nuestro cerebro o por la rotura de un vaso cerebral, el primer caso se denomina ictus isquémico que son más frecuentes afectando a un $85 \%$ del total cuya consecuencia final es el infarto cerebral; en el segundo caso nos referimos a ictus hemorrágico que son menos frecuentes pero su mortalidad es mayor(3).

Uno de los temas importantes al momento de la atención e intervención odontológica de un paciente que ha padecido ictus es la facilidad del sangrado ante los procedimientos que se le puedan efectuar, debido a que se encuentra bajo una terapia antiagregante plaquetaria, para prevenir trombosis arteriales y venosas en pacientes con historia de accidente cerebrovascular, los fármacos más usados son: ácido acetilsalicílico, clopidrogel y ticlopidina ${ }^{(4)}$. Cuando estos pacientes deben someterse a una intervención quirúrgica se plantea la necesidad de retirar la terapia antiagregante plaquetaria y someterlos a un posible incremento de riesgo al presentar una complicación tromboembolicacardiovascular o por el contrario, si se mantiene el tratamiento, podría aumentar el riesgo de presentar alguna complicación hemorrágica durante y/o después de la intervención(5).

En el protocolo odontológico convencional, en primera instancia se debe consultar al médico tratante para determinar si es seguro suspender el tratamiento por unos días, si se acepta la suspensión se realizará 3 o 4 días antes del procedimiento, tiempo requerido para se forme un numero de plaquetas adecuadas para reaccionar y realizar una adecuada hemostasia; durante el procedimiento se debe contar con medidas que promuevan la formación de coágulo estable y en caso de que no se presente sangrado postoperatorio es necesario restaurar la toma de medicamentos un día después del procedimiento(6). En caso de que no sea seguro suspender el tratamiento antiagregante plaquetario, existen otros protocolos alternativos que consisten en manejar los tratamientos de tipo quirúrgico con hemostáticos locales debido a que el sangrado es controlable; en el área dental, especialmente en cirugía maxilofacial, existen evidencias publicadas sobre éste manejo donde se ha demostrado un bajo número de complicaciones asociadas con extracciones $\mathrm{u}$ otras cirugías menores de la cavidad oral, sin suspender los fármacos antitrombóticos y tomando algunas precauciones mínimas ${ }^{(7)}$.

En este artículo se reporta un caso de paciente con antecedentes de ictus hace 4 años sometido a terapia antiagregante plaquetaria, el cual necesitaba rehabilitarse debido a pérdida de piezas dentarias con anterioridad. Se le realizaron los exámenes preoperatorios indicados y se le brindó un plan de tratamiento al cual accedió a 
realizarse, se tuvo en cuenta todas las consideraciones del caso manejo pre y post operatorio.

\section{Caso clínico}

Se presenta caso de paciente de 54 años de edad de sexo masculino que acude al máster de Odontología Integrada de Adultos y Pacientes Especiales de la Universidad de Sevilla cuyo motivo de consulta era la rehabilitación de piezas dentarias faltantes, se realizó anamnesis donde presentó antecedentes personales de ictus hace 4 años, antecedentes familiares de diabetes por parte de su padre e hipertensión y enfermedad cardiovascular por parte de su madre, actualmente padece de hipertensión ocular e hipercolesterolemia, medicado con Adiro 100mg (Antiagregante), clopidogrel 75mg (Antiagregante), ranitidina $300 \mathrm{mg}$ y simvastatina $40 \mathrm{mg}$ para la hipercolesterolemia.

El paciente refiere no presentar hábitos tóxicos como tabaquismo o ingesta de alcohol. Este paciente fue clasificado como ASA II. A la exploración extraoral se evidenció paciente normocefálico, implantación capilar androide con tercios proporcionales, piel perioral sana y perfil recto, sin alteraciones relevantes. A la exploración intraoral presentaba ausencia de órganos dentarios 21, 22, 23, 24, 26, 28, 15, 16, 18, 45, 46, 48, 36, 38 (Figura 1).

Atrición dental en órganos anterosuperiores y anteroinferiores (Figura 2), al examen periodontal presentó recesiones gingivales en órganos dentarios 14, 17, 23, 25, 27, 33, 35, 41, 44, 47, sangrado en 34, 27 y 13, no se evidenciaron bolsas periodontales, lesiones de furca ni movilidad. Se procedió a realizar montaje en articulador con el fin de analizar oclusión del paciente y realizar un plan de tratamiento el cual consistió en rehabilitación con prótesis fija completa superior e implantes para reemplazar órganos dentarios 36 y 46.

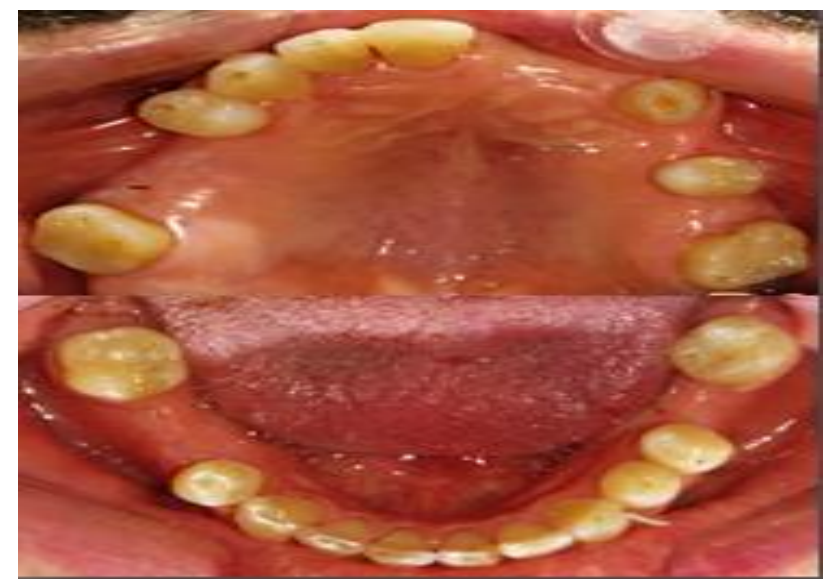

Figura 1. Paciente en cita inicial- exploración intraoral: Se observa en la exploración intraoral ausencia de órganos dentarios

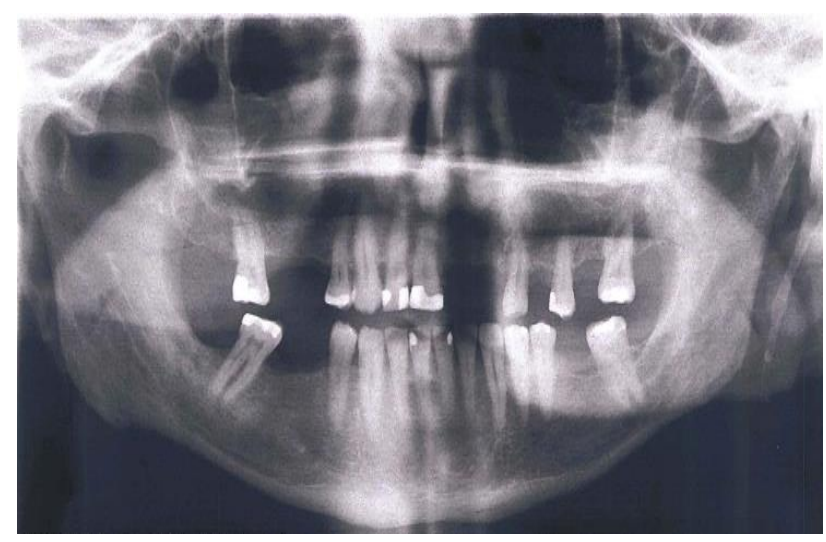

Figura 2. Ayuda diagnóstica - ortopantomografia inicial: Se observa atrición dental en órganos anterosuperiores y anteroinferiores

Se inició el tratamiento con la prótesis fija completa superior en forma de herradura realizando tallado de las piezas dentarias superiores sin endodoncia previa, utilizando anestesia local mepivacaina sin vasoconstrictor, colocando provisionales posteriormente; luego se continuó a tomar impresión definitiva para realizar con el laboratorio la estructura metálica posteriormente realizando prueba de ésta a la siguiente cita, se realizó cementación final de la prótesis realizando una rehabilitación completa definitiva en el sector superior (Figura 3). 


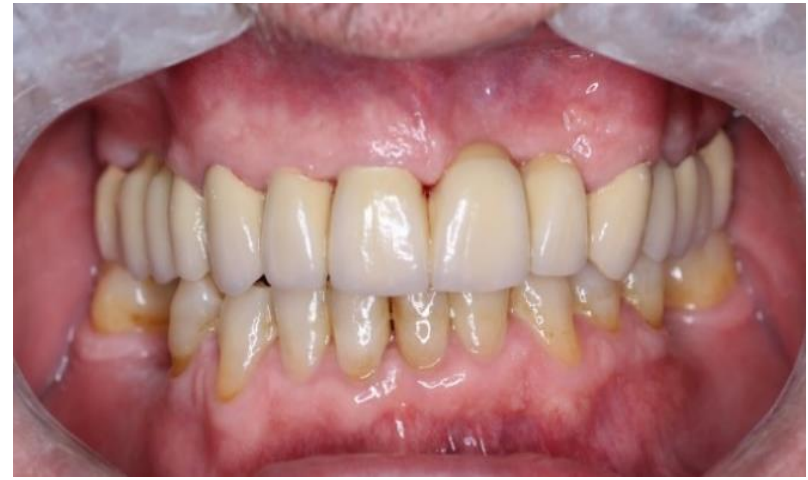

Figura 3. Trabajo prostodontico superior

finalizado: Se observa rehabilitación completa definitiva del sector superior con prótesis parcial fija

Para la colocación de implantes en sector inferior, con el fin de reemplazar los órganos dentarios 36, 45 y 46, se inició realizando un TAC (tomografía axial computarizada), para evaluar el espesor y densidad ósea; el examen arrojó que existía un buen soporte de hueso para los implantes. Antes de la cirugía se realizó interconsulta con el médico tratante de la patología de base y se suspendió el clopidrogel 75 mg cuatro días antes y se mantuvo el Adiro $100 \mathrm{mg}$. El procedimiento se inició en la hemiarcada inferior izquierda para reemplazar órgano dentario 36, colocándose anestesia local (mepivacaina ${ }^{\circledR}$ al $3 \%$ sin vasoconstrictor), se realizó incisión supracrestal e intrasulcular con bisturí convencional (Bard Parker ${ }^{\circledR} \mathrm{N}^{\circ} 3$, hoja $\mathrm{N}^{\circ}$ 15) para colocar implante STRAUMAN stándar plus de $4,1 \mathrm{~mm} \times 10 \mathrm{~mm}$ de longitud roxolid SLActive; con ayuda de una guía quirúrgica, se inició la perforación con fresa de diamante redonda de 2,3 $\mathrm{mm}$, luego con fresa piloto de 1,2 $\mathrm{mm}$, luego se utilizó la fresa helicoidal $3,5 \mathrm{~mm}$ y por último la fresa de perfil, todas a $10 \mathrm{~mm}$ de longitud. No se presentó abundante sangrado durante el procedimiento. Se colocó sutura de dos puntos simples nylon negro monofilamento $4 / 0$ $\left(\right.$ Ethilon $^{\mathrm{TM}}$ ) no reabsorbibles, la cual se retiró a la semana sin complicaciones (Figura 4).

En el postoperatorio se formuló amoxicilina cápsulas de $1 \mathrm{~g}$ cada 12 horas durante 7 días, y en caso de dolor paracetamol tabletas de $500 \mathrm{mg}$ cada 6 horas. Las recomendaciones y la prescripción farmacológica se dieron por escrito. El paciente volvió a tomar el clopidrogel dos días posteriores a la cirugía. Se realizó control a las dos semanas presentando correcta cicatrización y buen resultado funcional y estético de todo el tratamiento (Figura 5).

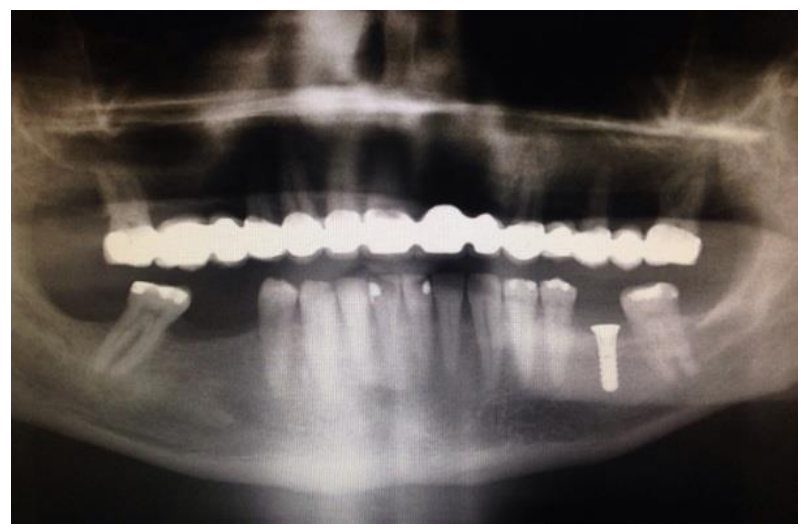

Figura 4. Ortopantomografía final: Se observa la correcta posición del implante dental Strauman con adecuado paralelismo y distancia con el od\#36

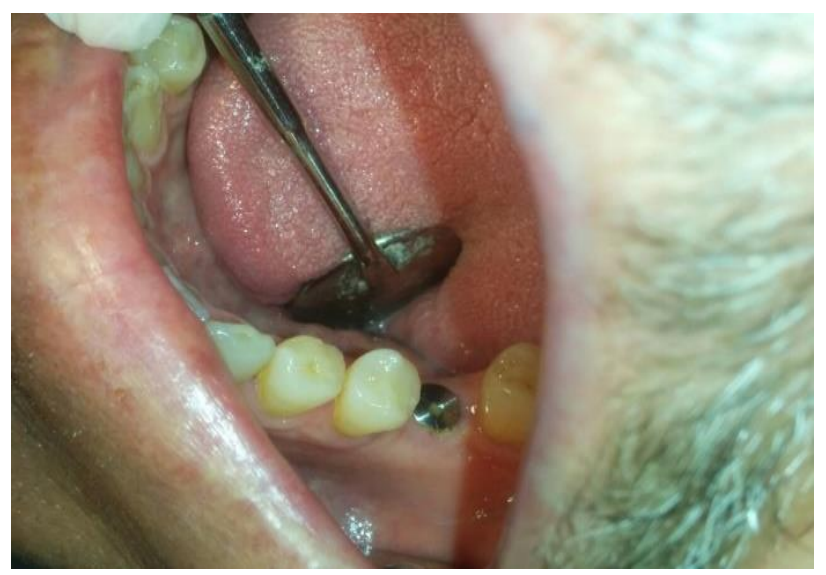

Figura 5. Control postoperatorio del implante: Se observa control postoperatorio buena respuesta de los tejidos y acople del implante

\section{Discusión}

Actualmente es frecuente encontrarse con pacientes con antecedentes de ictius en la consulta odontológica, por esto, saber manejarlos se ha vuelto fundamental para el éxito de cualquier tratamiento. Se ha determinado un manejo pre, peri y postoperatorio; en el preoperatorio se hace énfasis en realizar una completa y exhaustiva anamnesis donde se determine la patología de base, antecedentes 
hemorrágicos, medicamentos administrados para evaluar las posibles interacciones farmacológicas y otras condiciones sistémicas que aumenten el tiempo de sangría, como enfermedades hepáticas o alcoholismo ${ }^{(8)}$. Es fundamental la interconsulta con el médico tratante en caso de terapia dual, para determinar la suspensión o no de uno o varios medicamentos, indicación de exámenes serológicos; se debe planificar la cirugía en las primeras horas de la mañana y si es posible los primeros días de la semana para poder resolver eventos hemorrágicos postoperatorios y realizar la toma de presión arterial(9).

En el perioperatorio es recomendable realizar los procedimientos en un área limitada de la cavidad oral (por cuadrantes), las punciones anestésicas deben ser hechas de manera cuidadosa utilizando técnica de aspiración y de ser posible evitando la colocación de anestesia troncular, utilizar sutura reabsorbible sin tensión, los procedimientos deben ser lo más atraumaticos posibles, la utilización de tapones hemostáticos tales como esponjas de gelatina reabsorbible, colágeno o fibrina y cera de hueso deben ser imprescindibles(10).

En el postoperatorio es importante tomar la presión arterial, evaluar al paciente por lo menos una hora antes de ser dado de alta, entregar por escrito las indicaciones postoperatorias habituales después de una cirugía bucal, saber que el único analgésico que se puede recetar es el acetaminofén o paracetamol debido a que los antiinflamatorios no esteroides (AINES) aumentan los riesgos de hemorragias(11).

Brennan et al., introdujeron nuevas recomendaciones en el manejo de estos pacientes que consiste en mantener la terapia antiagregante antes de los procedimientos como exodoncias debido a que el sangrado que puede presentarse durante el acto quirúrgico puede controlarse con medidas hemostáticas eficientes como comprensión mecánica con gasa, celulosa oxidada, esponja de gelatina, entre otras y que no se van a producir problemas hemorrágicos a menos que el tiempo de sangría se encuentre muy prolongado(12).
No existe evidencia científica en cuanto a complicaciones hemorrágicas postoperatorias en pacientes con terapia antiplaquetaria (TAP) que van a ser sometidos a cirugías orales; sin embargo es posible la pérdida de la función antitrombótica si es suspendida la TAP y sus consecuencias cardiovasculares(13). En otro estudio se comparó la función plaquetaria en pacientes que se encuentran bajo tratamiento con ácido acetilsalicílico por más de 6 meses con un grupo control sano, se pudo concluir que el $80 \%$ de los pacientes bajo TAP mostraron una función plaquetaria disminuida, presentando mayor hemorragia intraoperatoria que el grupo control, la cual fue controlable a través de métodos hemostáticos locales debido a que la hemorragia no se produjo de manera excesiva(14). Una de las mayores ventajas de no suspender la TAP es evitar complicaciones cardíacas mayores. Además, se produce una disminución significativa del riesgo de eventos adversos cerca de 10 días después de la suspensión de los medicamentos(15).

En el caso reportado no se suspendió el Adiro pero si el clopidrogel debido a que se siguieron las indicaciones del médico tratante y no existieron complicaciones a nivel sistémico. Sin embargo, los nuevos estudios e indicaciones para cirugía nos dan una visión más amplia y actualizada del manejo para este tipo de pacientes brindándonos alternativas más eficientes con el fin de evitar complicaciones mayores.

\section{Conclusión}

Es fundamental realizar una correcta historia clínica y las pruebas de laboratorio necesarias para predecir alteraciones hemostáticas que puedan interferir con el tratamiento a realizar, sobre todo si es de tipo quirúrgico. Los pacientes sometidos a TAP pueden ser tratados con o sin modificación de su tratamiento, siempre y cuando, se realice la interconsulta con el médico tratante, además de tener conocimiento de sus enfermedades de base, estilos de vida saludables y contar con las medidas hemostáticas para realizar intervenciones quirúrgicas sin complicaciones para así poder tener un éxito del tratamiento odontológico en estos pacientes. 


\section{Agradecimientos}

Los autores agradecen a la Universidad de Sevilla y al Máster de Odontología integrada de adultos y pacientes especiales por el espacio brindado para la realización de este caso.

\section{Conflicto de intereses}

Los autores no reportan conflicto de interés.

\section{Referencias}

1. Aleman JA, Urioste LMR, Hernández ML, García PA, López GT, Claros NM. Control de los factores de riesgo cardiovascular en pacientes con ictus atendidos en Atención Primaria en España. Estudio ICTUSCARE. Medicina clínica, 2011;136(8), 329-335.

2. Rubio-Alonso LJ, Martínez-Rodríguez N, CáceresMadroño E, Fernández-Cáliz F, Martínez-González JM. Protocolos de actuación con la exodoncia en pacientes geriátricos antiagregados y anticoagulados, Avances en Odontoestomatología. 2015;31(3):203-214.

3. Arias-Rivas S, Vivancos-Mora J, Castillo J. Epidemiología de los subtipos de ictus en pacientes hospitalizados atendidos por neurólogos: resultados del registro EPICES (I). Rev Neurol, 2012;54(7):385-393.

4. Cedeño JA, Rivas N, Tuliano RA. Manejo odontológico en pacientes con terapia antiagregante plaquetaria. Revista Odontológica Mexicana. 2013;17(4):256-260.

5. Wright L, Hill KM, Bernhardt J, Lindley R, Ada L, Bajorek BV, Nelson MR. Stroke management: updated recommendations for treatment along the care continuum. Internal Medicine Journal. 2012;42(5):562569.

6. Fernández-Tresguerres FG, Sirvent AA, Denche JTG, Fernández I. Nuevos anticoagulantes orales: repercusión odontológica. Científica dental: Revista científica de formación continuada, 2016:13(2), 59-68.

7. Johnston SA. New generation of antiplatelet and anticoagulant medication and the implications for the dental surgeon. Dental Update. 2015;42(9):840-854.

8. Murphy J, Twohig E, McWilliams SR. Dentists' approach to patients on anti-platelet agents and warfarin: a survey of practice. Journal of the Irish Dental Association. 2010;56(1):28-31

9. Martínez SR, Serna JT, Silvestre FJ. Manejo odontológico en el paciente cirrótico. Gastroenterología y Hepatología, 2016:39(3), 224-232.

10. Ripollés-de-Ramón J, Muñoz-Corcuera M, Bravo-Llatas C, Bascones-Martínez A. Aplicación de un gel de ácido tranexámico en pacientes tratados con anticoagulantes orales. Medicina Clínica. 2014;143(11):484-488

11. Otiniano ALV, Gómez PAM. Manejo odontológico de pacientes con tratamiento anticoagulante. In Crescendo Ciencias de la salud. 2016;2(2):307-313.

12. Brennan MT, Wynn RL, Miller CS. Aspirin and bleeding in dentistry: an update and recommendations. Oral Surgery, Oral Medicine, Oral Pathology, Oral Radiology, and Endodontology. 2007;104(3):316-323.
13. Carrizo A, Carrasco D. Oral surgery in patients under antithrombotic therapy. Narrative review. Journal of Oral Research. 2015;4(1):58-64.

14. Kwok C, McIntyre A, Janzen S, Mays R, Teasell R. Oral care post stroke: a scoping review. Journal of oral Rehabilitation. 2015;42(1):65-74.

15. Motovska Z. Management of antiplatelet therapy in patients at risk for coronary stent thrombosis undergoing non-cardiac surgery. Drugs. 2011;71(14):1797-1806. 